\title{
Avaliação do risco de extinção das Urticineae das restingas do estado do Rio de Janeiro
}

Assessment of extinction risk of Urticineae from sandy coastal plains of the State of Rio de Janeiro

\author{
Leandro Cardoso Pederneiras ${ }^{1,3}$, Andrea Ferreira da Costa $^{1}$, Jorge Pedro Pereira Carauta ${ }^{\dagger 1}$ \\ \& Sergio Romaniuc Neto ${ }^{2}$
}

\begin{abstract}
Resumo
Foram realizados o georreferenciamento e as análises de avaliação de risco de extinção seguindo os critérios e categorias da IUCN para 32 espécies de Urticineae (Cannabaceae, Ulmaceae, Urticaceae e Moraceae) ocorrentes nas restingas do estado do Rio de Janeiro. Materiais correspondentes a essas espécies foram examinados nos principais herbários brasileiros, sendo selecionados 2524 registros para a avaliação. Dentre as 32 espécies, Ampelocera glabra, Celtis spinosa, Ficus cyclophylla, F.nevesiae, Maclura brasiliensis e Phyllostylon brasiliense encontraram-se ameaçadas de extinção, já Brosimum guianense, Dorstenia arifolia, Ficus castellviana, F.pulchella, Sorocea guilleminiana e S. hilarii deixaram de ser consideradas ameaçadas de extinção pelo recente estudo. Os resultados apontaram, como localidades de importância conservacionista, os municípios de Armação de Búzios, Cabo Frio, Rio de Janeiro e Saquarema.
\end{abstract}

Palavra-chave: Moraceae, Urticaceae, Ulmaceae, Cannabaceae.

\begin{abstract}
This paper conducted georeferencing and analyzes of extinction risk assessments following the criteria and categories from IUCN for 32 species of Urticineae (Cannabaceae, Ulmaceae, Moraceae and Urticaceae) occurring in the sandy coastal plains of the state of Rio de Janeiro. Materials corresponding to these species were examined in major Brazilian herbaria and 2524 records were selected for evaluation. Among the 32 species, Ampelocera glabra, Celtis spinosa, Ficus cyclophylla, F.nevesiae, Maclura brasiliensis and Phyllostylon brasiliense fall into endangered category, and Brosimum guianense, Dorstenia arifolia, Ficus castellviana, F.pulchella, Sorocea guilleminiana and S. hilarii were not considered threatened. The locations of conservation importance belong to the municipalities of Armação de Búzios, Cabo Frio, Rio de Janeiro and Saquarema.
\end{abstract}

Key word: Moraceae, Urticaceae, Ulmaceae, Cannabaceae.

\section{Introdução}

A subordem Urticineae é um grupo de plantas que pertencem à ordem Rosales e reúnem as famílias Cannabaceae, Moraceae, Ulmaceae e Urticaceae. São pantropicais e caracterizadas principalmente por possuírem cistólitos, flores inconspícuas, floema estratificado, dois carpelos, ovário unilocular com único óvulo apical e pólen porado (Judd et al. 2007). No Brasil, Urticineae está representada por 312 espécies (36 gêneros), distribuidas principalmente nas florestas ombrófilas Atlânticas e Amazônicas (Romaniuc-Neto et al. 2012a,b; Romaniuc-Neto \& Gaglioti 2012; Romaniuc-Neto \& Torres 2012).
Urticineae tem recebido contribuições progressivas em relação a conservação, baseado nos critérios da IUCN. A União Internacional de Conservação da Natureza - IUCN, estabeleceu em 1994 critérios para avaliar o estado de conservação das espécies com nove categorias (Extinta, Extinta na natureza, Criticamente em perigo, Em perigo, Vulnerável, Quase ameaçada, Menos preocupante, Deficiente de dados e Não avaliada) visando unificar e padronizar as avaliações mundiais (IUCN 1994). Em Moraceae, o principal trabalho de avaliação das espécies brasileiras abordou 257 espécies (sensu lato) com 83 ameaçadas de extinção, uma listagem

\footnotetext{
${ }^{1}$ Universidade Federal do Rio de Janeiro, Depto. Botânica, Museu Nacional, 20940-040, São Cristóvão, Rio de Janeiro, RJ, Brazil.

${ }^{2}$ Instituto de Botânica, Herbário, C.P. 3005, 01031970, São Paulo, SP, Brazil.

${ }^{3}$ Autor para correspondência: leandro.pederneiras@gmail.com
} 
minuciosa resultado da longa vivência de campo dos pesquisadores envolvidos (Carauta et al. 1996). No estado do Rio de Janeiro, Carauta (1996) listou 34 espécies ameaçadas de extinção. Ambos os trabalhos seguiram os critérios e categorias da IUCN (1994). Na lista oficial das espécies da flora brasileira ameaçadas de extinção somente quatro foram consideradas (MMA 2008) e outras 17 na lista vermelha da IUCN (IUCN 2012). Trabalhos baseados em dados locais e regionais são importantes para a tomada de decisões para a manutenção da biodiversidade dentro do próprio espaço de análise (e.g. Carauta 1996; Pederneiras et al. 2011a,b; Pelissari \& Romaniuc 2013). Por outro lado, a avaliação o estado de conservação da espécie como um todo (nacional ou globalmente) muda o foco para a sobrevivência do táxon.

A vegetação das restingas fluminenses está sofrendo intensa perda de hábitat principalmente pela especulação imobiliária, o que torna urgente o conhecimento do estado de conservação de suas espécies. A partir da análise regional de risco de extinção das espécies de Urticineae ocorrentes nas restingas fluminenses (Pederneiras et al. 2011a,b), procurou-se ampliar a análise para uma avaliação nacional com o objetivo de contribuir para o provimento de listas vermelhas e para destacar localidades de importância conservacionista para as espécies de Urticineae.

\section{Material e Métodos}

Esse trabalho foi baseado em um exame taxonômico acurado das espécies dos herbários do Rio de Janeiro (R, RB, GUA e HB), São Paulo (SP e SPF), Bahia (HUEFS, CEPEC, ALCB, HRB) e Manaus (INPA), sendo selecionados 2524 registros para a fase de análise de dados de localidade de ocorrência que foram georreferenciados e mapeados para o auxílio na avaliação do risco de extinção.

Foram utilizados os critérios e categorias da IUCN (Versão 9.0, 2011) e recomendações de Borges et al. (2012). Para área de ocupação foi utilizada a escala recomendada de $4 \mathrm{~km}^{2}$ por célula. Para medições de extensão de ocorrência foi utilizado o Google Earth (Google Earth ${ }^{\mathrm{TM}}$ serviço de mapa) e para cálculo de áreas foi utilizado o software Google Earth Path (Sgrillo 2012).

Nas espécies classificadas como ameaçadas de extinção (Vulnerável e Em Perigo) foram selecionados e citados todos os materiais de interesse para uma avaliação conservacionista e apenas materiais em localidades repetidas foram excluídos. Foi inferido se as localidades de ocorrência das espécimes encontram-se ainda em estado conservado, ou não, para a contagem do critério B2a da IUCN. Localidades que provavelmente encontram-se degradadas não foram contadas.

Para as espécies consideradas não ameaçadas (LC, NT) foi selecionado e citado um material de herbário (geralmente o que foi coletado mais recentemente) para cada estado do Brasil, com finalidade de testemunhar a abrangência e justificar a categorização.

As espécies foram georreferenciadas na própria exsicata e mapeadas no programa Arcview (ENRI 1999), mas somente os mapas das espécies consideradas ameaçadas de extinção, tanto pelo presente trabalho como pelos estudos prévios (Carauta et al. 1996; Carauta 2002; MMA 2008; IUCN 2012), foram consideradas para fins de categorização.

\section{Resultados}

Das 32 espécies avaliadas e categorizadas no presente trabalho, seis estão ameaçadas de extinção (Ampelocera glabra Kuhlm., Celtis spinosa Spreng., Ficus cyclophylla (Miq.) Miq., F.nevesiae Carauta, Maclura brasiliensis (Mart.) Endl. e Phyllostylon brasiliense Capan.) e 26 em menor preocupação (Tab. 1). Todas as mudanças de categoria ocorreram em razão da ampliação do conhecimento através das novas coletas e informações de campo dos últimos 15 anos.

\section{Espécies ameaçadas de extinção}

1.1 Ampelocera glabra Kuhlm. Fig. 1a

Possui $28 \mathrm{~km}^{2}$ de área, com subpopulações severamente fragmentadas inferidas pelas análises de material de herbário que totalizaram apenas três coletas nessas três últimas décadas (vide material selecionado abaixo). Além disso, foi observado que a área da coleta G.S.Z.Rezende 69 vem sofrendo desmatamento para ocupação humana. EN B2ab(iii,iv).

Material selecionado: BAHIA: Itaibó, estrada para Apuarema, 28.X.1970, T.S.Santos 1210 (CEPEC, RB). Jussari, Reserva Particular do Patrimônio Natural Serra do Teimoso, 8.IX.1998, A.M.Amorim et al. 2619 (SP). ESPÍRITO SANTO: Rio Doce, Goitacazes, 15.XI.1943, J.G.Kuhlmann 6429 (RB, CEPEC). MINAS GERAIS: Rio Doce, Fazenda Paraiso, 13.X.1997, E.L.M.Catharino 2172 (SP). RIO DE JANEIRO: Cabo Frio, Condomínio Florestinha, 6.XII.2001, G.S.Z.Rezende et al. 69 (RB). Rio de Janeiro, Laranjeiras, Reserva Florestal da Fábrica Aliança, 8.XI.1922, J.G.Kuhlmann 6794 (RB). Horto Florestal, 23.XII.1926, J.G.Kuhlmann 1221 (RB). Poses, Avellar, G.M.Nunes 80 (RB). 
Tabela 1 - Categorias de conservação das espécies de acordo com a IUCN. Categorias: DD: data deficient (dados deficientes); LC: least concern (menor preocupação); VU: vulnerable (vulnerável); EN: endangered (em perigo); NT: near threatened (próximo à ameaçada); NE: not evaluated (não avaliada). Dados do Brasil baseado em Carauta et al. (1996), Carauta (2002).

Table 1 - Conservation status of species according to IUCN. Categories: DD: data deficient; LC: least concern; VU: vulnerable, EN: endangered, NT: near threatened, NE: not evaluated. Data from Brazil based on Carauta et al. (1996) and Carauta (2002).

\section{Espécies}

Ampelocera glabra Kuhlm.

Brosimum guianense (Aubl.) Huber

Cecropia glaziovii Snethl.

Cecropia lyratiloba Miq.

Celtis brasiliensis (Gardner) Planch.

Celtis iguanaea (Jacq.) Sarg.

Celtis spinosa Spreng.

Coussapoa microcarpa (Schott) Rizzini

Dorstenia arifolia Lam.

Dorstenia cayapia Vell.

Ficus arpazusa Casar.

Ficus castellviana Dugand

Ficus clusiifolia (Miq.) Schott

Ficus cyclophylla (Miq.) Miq.

Ficus eximia Schott

Ficus gomelleira Kunth

Ficus hirsuta Schott

Ficus luschnathiana (Miq.) Miq.

Ficus maximiliana (Miq.) Mart.

Ficus nevesiae Carauta

Ficus organensis (Miq.) Miq.

Ficus pulchella Schott ex Spreng.

Ficus trigona L.f.

Laportea aestuans (L.) Chew

Maclura brasiliensis (Mart.) Endl.

Maclura tinctoria (L.) D. Don ex Steud.

Phyllostylon brasiliense Capan.

Sorocea guilleminiana Gaudich.

Sorocea hilarii Gaudich.

Trema micrantha (L.) Blume

Urera aurantiaca Wedd.
MMA IUCN Presente 20082010 pesquisa

EN

VU

LC

NE

LC

NE

LC

LC

LC

EN

LC

LC

VU

DD

LC

NT

DD

LC

LC

LC

VU

LC LC

LC

LC

EN

EN VU

NT

LC

NT

LC

NT

NT LC

LC

LC

LC

VU

VU

NT

LC

VU

VU LC

LC

LC

LC

EN

NT

LC EN

LC

EN

VU

VU LC

EN 


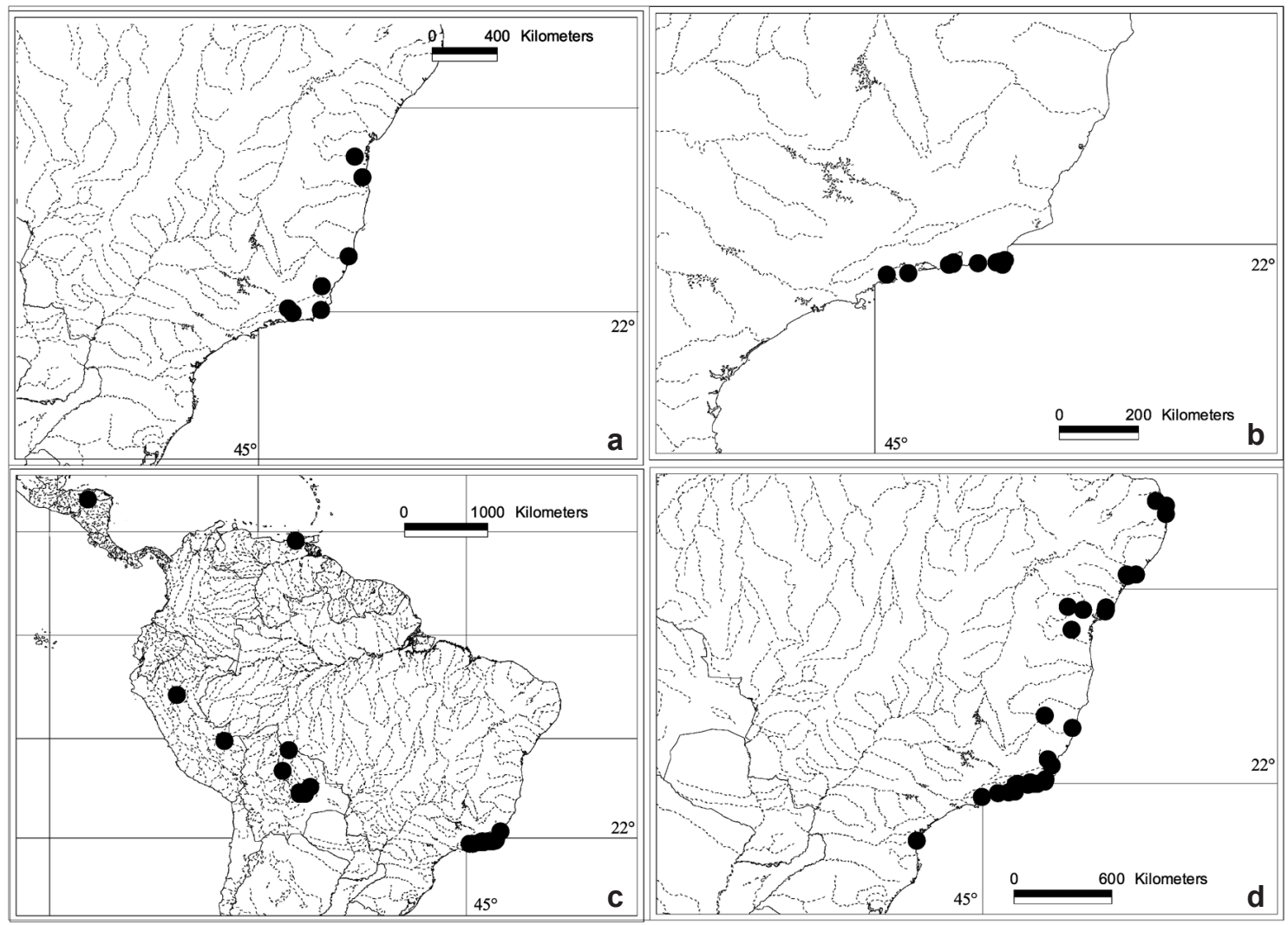

Figura 1 - Mapas de distribuição geográfica - a. Ampelocera glabra; b. Ficus nevesiae; c. Maclura brasiliensis; d. Ficus cyclophylla.

Figure 1 - Geographic distribution maps - a. Ampelocera glabra; b. Ficus nevesiae; c. Maclura brasiliensis; d. Ficus cyclophylla.

\subsection{Celtis spinosa Spreng.}

Fig. 2a

No Brasil, possui uma área de ocupação de ca. $20 \mathrm{~km}^{2}$, com subpopulações severamente fragmentadas inferidas pelas análises de material de herbário que totalizaram apenas duas coletas nessas três últimas décadas (vide material selecionado abaixo). As duas localidades encontradas no Rio de Janeiro, estão dentro de unidades de conservação, mas que sofrem constante degradação ambiental pela pressão imobiliária e turística do entorno, pela falta de controle e vigilância permanente, e pela falta de instalações físicas e infraestruturas próprias de unidades de conservação. EN B2ab(iii,iv)

Material selecionado: ARGENTINA: Buenos Aires, La Plata, 2.XII.1971, J.P.P.Carauta 1417 (GUA); BRASIL. MATO GROSSO: Cuiabá, 1914, J.G.Kuhlmann 155 (R); RIO GRANDE DO SUL: Taim, Vila do Taim, 17.X.1979, D.Araujo 1285 (GUA). RIO DE JANEIRO: Niterói, Alto Mourão, 9.IX.1982, Andreata et al. 516 (GUA). Saquarema, Reserva Ecológica Estadual de Jacarepiá, 29.X.1991, D.Araujo et C.Farney 9474 (GUA).
1.3 Ficus cyclophylla (Miq.) Miq. $\quad$ Fig. 1d Esta espécie ocorre na Floresta Ombrófila Densa Atlântica, da Paraíba até São Paulo, mais particularmente em Matas de Restinga. Supondo que cada geração dure pelo menos ca. 30 anos (Carauta 1989), estima-se um declínio de pelo menos $30 \%$ em três gerações, ou 90 anos, agravado pela alta valorização imobiliária das regiões litorâneas que tente a pressionar os últimos refúgios de matas de restingas e a perda de área por construções. VU A2c

Material selecionado: BAHIA: Camaçari, 10.VI.2006, M.L.Guedes 12162 (ALCB). ESPÍRITO SANTO: Linhares, 7.VI.1994, D.A.Folli 2322 (GUA). MINAS GERAIS: Governador Valadares, 23.II.2004, R.M.Castro et Teixeira 917 (HUEFS). PARAÍBA: Araruna, 14.IV.2002, M.R.Barbosa et al. 2415 (HUEFS). RIO DE JANEIRO: Saquarema, Jacarepiá, 11.IV.2008, L.C.Pederneiras 414 (R). SÃO PAULO: Ubatuba, 14.XI.1993, M.D.de Moraes et al. 29295 (SPF). SERGIPE: Pirambú, 28.I.1992, C.Farney et al. 2976 (RB). 


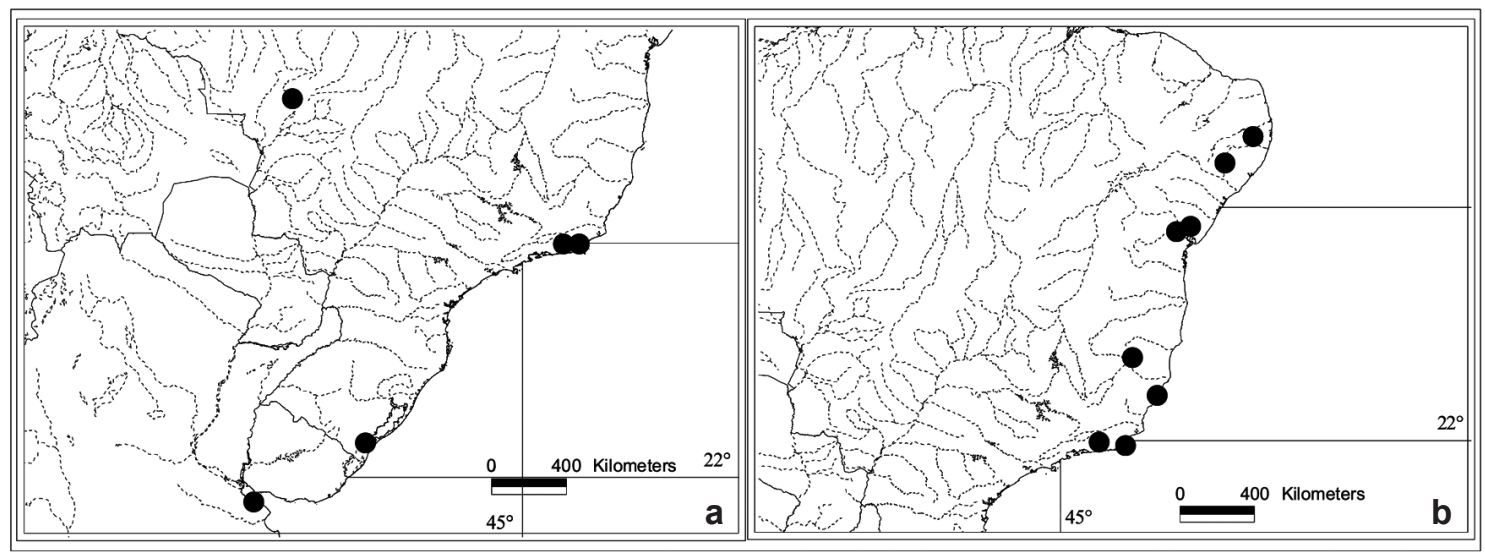

Figura 2 - Mapas de distribuição geográfica - a. Celtis spinosa; b. Phyllostylon brasiliense.

Figure 2 - Geographic distribution maps - a. Celtis spinosa; b. Phyllostylon brasiliense.

\subsection{Ficus nevesiae Carauta}

Fig. 1b

Esta espécie possui uma área de ocorrência de ca. $24 \mathrm{~km}^{2}$, com subpopulações severamente fragmentadas inferidas pelas análises de material de herbário que totalizaram apenas sete coletas reconhecidas. A maioria dos indivíduos conhecidos estão dentro de unidades de conservação no Rio de Janeiro, mas sob constante ameaça ambiental, principalmente pela pressão imobiliária e turística do entorno, pela falta de controle e vigilância permanente das áreas, e pela falta de instalações físicas e infraestruturas próprias de unidades de conservação. VU B2ab(i,ii,iii,iv).

Material examinado: RIO DE JANEIRO: Reserva Biológica Estadual da Praia do Sul, 16.II.1984, D.Araujo 6126 (RBR). Arraial do Cabo, Restinga de Massambaba, 4.III.2008, L.C.Pederneiras et al. 383, 385 (R). Botafogo, 12.XI.2000, B.E.Diaz 395 (GUA). Cabo Frio, Peró,14.IX.2002, A.Quinet et al. 729 (RB). Parati, 28.IX.1996, R.Mello-Silva \& Santos 1250 (SPF). Saquarema, Jaconé, 8.IV.1992, D.Araujo 9610 (GUA). Lagoa de Jacarepaguá, 8.VI.1987, H.C.Lima et al. 6523 (RB). Prainha, 2.IV.2011, L.C.Pederneiras et A.G.Carvalho $672(\mathrm{R})$.

1.5 Maclura brasiliensis (Mart.) Endl. Fig. 1c

Apesar da extensão de ocorrência neotropical (Kaastra 1973), essa espécie possui uma área de ocupação de ca. $52 \mathrm{~km}^{2}$, com subpopulações severamente fragmentadas inferidas pelas análises de material de herbário que totalizaram apenas quatro localidades conhecidas. As populações entre o Brasil e a Bolívia estão isoladas em mais de $2000 \mathrm{~km}$. No Brasil, atualmente só ocorre em Armação de Búzios, um refúgio de clima estacional semidecidual no Rio de Janeiro que, nessa pesquisa, fica caracterizado como uma "location" pela forte pressão imobiliária e turística que ameaça a perda de espaço dessa espécie na região. Em Campos dos Goitacazes foram feitas excursões de coleta (nos anos de 2007-2009) nas proximidades do complexo lagunar Grussaí e Iquiparí, únicos remanescentes de vegetação de restinga restante na região, mas não foi reencontrada. EN B2ab(ii,iii,iv).

Material selecionado: BRASIL. RIO DE JANEIRO: Armação de Búzios, Praia da Gorda, 17.XII.1998, A.Q.Lobão et al. 395 (RB). Praia da Tartaruga, 12.XI.1998, A.Q.Lobão et al. 381 (RB). Campos dos Goitacazes, M.A.P.Wied-Neuwied (U, BR). BOLÍVIA. Santa Cruz, Andrés Ibáñez, 17.XII.1991, M.Nee 42145 (RB). HONDURAS. Olancho, vizinhanças de Catacamas, 18.III.1949, C.Standley 18431 (US). PERU. Madre de Dios, Manu, 11.IX.1986, R.B.Foster 11354 (F).

1.6 Phyllostylon brasiliense Capan. $\quad$ Fig. $2 \mathrm{~b}$

Essa espécie ocorre do Brasil até a América Central (Todzia 2001; CONABIO 2009), mas outros trabalhos consideram endêmicas da costa Atlântica brasileira (Todzia 1992; Pederneiras et al. 2011b). Se for considerado somente o Brasil, possui uma área de ocupação de apenas ca. $24 \mathrm{~km}^{2}$, com subpopulações severamente fragmentadas inferidas pelas análises de material de herbário que totalizaram apenas três coletas nessas três últimas décadas. No Rio de Janeiro, ocorre em Armação de Búzios, considerado uma "location" pelas mesmas considerações citadas em Maclura brasiliensis, já em São Cristóvão, a área que Glaziou coletou pela primeira vez, foi completamente suprimida pelas construções portuárias. EN B2ab(ii,iii,iv) 
Material examinado: BAHIA: Itatim, Morro das Tocas, 25.XI.1995, F.França et al. 1432 (HUEFS); km 9 da estrada Serra Preta-Ipirá, 24.X.1970, Andrade-Lima 6190 (SP). ESPÍRITO SANTO: Victoria, Fazenda do Maruhype, 23.X.1930, J.G.Kuhlmann 485 (RB). MINAS GERAIS: Figueira (atualmente Governador Valadares), 25.IX.1929, J.G.Kuhlmann 251 (RB). PERNAMBUCO: Águas Belas, Serra do Cumunati, 21.XI.1969, AndradeLima 5616 (SP). Surubim, Salobra, 20.VII.1972, Andrade-Lima 6916 (SP). RIO DE JANEIRO: Armação de Búzios, Tucuns, 7.XI.1985, H.C.Lima et al. 5188 (RB). Rio de Janeiro, São Cristóvão, 28.X.1890, A.Glaziou (RB 6842).

Espécies anteriormente consideradas ameaçadas de extinção ou próximas

As espécies a seguir foram consideradas ameaçadas de extinção em estudos anteriores (Carauta et al. 1996; MMA 2008; IUCN 2010), mas, após o exame de material de herbário e mapeamento, uma ampliação e um refinamento do conhecimento foi alcançado, resultando numa mudança de categorização para menor preocupação (LC). Todas possuem coletas recentes tanto dentro quanto fora de Unidades de Conservação, além de extensão de ocorrência maior que $20 \mathrm{mil} \mathrm{km²}$.

\subsection{Brosimum guianense (Aubl.) Huber}

Fig. 3a

Extensão de ocorrência: ca. 4,7 milhões de $\mathrm{km}^{2}$. Material selecionado: ACRE: Plácido de Castro, 17.X.1980, B.Nelson 725 (RB). ALAGOAS: São Luis do Quitunde, 22.XI.2003, R.P.Lyra-Lemos 8117 (ALCB). AMAPÁ: Rio Araguari, 29.IX, I.M.Pires 51311 (R). AMAZONAS: Rio Apitua, 30.VI.1971, G.T.Prance et al. 13920 (R). BAHIA: Itaberaba, 18.I.2006, L.P.Queiroz 12062 (HUEFS). ESPÍRITO SANTO: Conceição da Barra, 21.IX.1993, O.J.Pereira 4902 (SP). MATO GROSSO: São Félix do Araguaia, 17.X.1985, Cid-Ferreira et al. 6506 (SPF). MATO GROSSO DO SUL: Bataguaçu, 22.XI.1992, I. Cordeiro et al. 1051 (SP). MINAS GERAIS: Jequeri, 27.XI.1997, A.Salino 3801 (GUA). PARÁ: Altamira, 25.X.1986, S.A.M.Souza 483 (HRB). PARAÍBA: Espirito Santo, Engenho São Paulo, 25.XI.1968, Andrade-Lima 5476 (SP). PERNAMBUCO: São Lourenço da Mata, 6.V.2000, K.Almeida \& Figueiredo 41 (CEPEC). RIO DE JANEIRO: Saquarema, 11.IV.2008, L.C.Pederneiras 407 (R). RONDÔNIA: Porto Velho, 19.IV.1987, M.Nee 34949 (SP). RORAIMA: Serra do Surucucu, 19.X.1991, S.Almeida \& Cordeiro 701 (HUEFS). SÃO PAULO: Sete Barras, 15.I.2004, N.M.Ivanauskas et al. 5016 (RB). SERGIPE: Estância, 28.XI.1993, A.M.Amorim et al. 1519 (R). TOCANTINS: Paraná, 10.IX.2003, A.C.Sevilha et al. 3506 (SP).

\subsection{Dorstenia arifolia Lam.}

Fig. $3 b$

Extensão de ocorrência: ca. 140 mil km².

Material selecionado: BAHIA: Itanhaém, 29.XII.2004, A.M.Amorim et al. 4583 (CEPEC).ESPÍRITO SANTO: Itarana, 26.II.2003, J.A.Lombardi et al. 5222 (SPF). MINAS GERAIS: Jequeri, 2.V.1998, A.Salino \& Morais 4290 (GUA). RIO DE JANEIRO: Grumari, 14.VII.2008, L.C.Pederneiras \& M.D.M.Vianna-Filho 471, 472 (R). SÃO PAULO: Caraguatatuba, 9.IX.2000, R.S.Bianchini et al. 1448 (SPF).

\subsection{Dorstenia cayapia Vell.}

Fig. 3c

Extensão de ocorrência: ca. 160 mil km².

Material selecionado: BAHIA: Itanhaém, 29.XII.2004, A.M.Amorim et al. 4583 (CEPEC). ESPÍRITO SANTO: Itarana, 26.II.2003, J.A.Lombardi et al. 5222 (SPF). MINAS GERAIS: Jequeri, 2.V.1998, A.Salino \& Morais 4290 (GUA). RIO DE JANEIRO: Campos, 17.V.2007, G.Heiden et al. 769 (RB). SÃO PAULO: Caraguatatuba, 9.IX.2000, R.S.Bianchini et al. 1448 (SPF).

\subsection{Ficus eximia Schott}

Fig. 3d

Extensão de ocorrência: ca. $230 \mathrm{mil} \mathrm{km²}$.

Material selecionado: BAHIA: Itatim, 29.I.1986, F.França et al. 1638 (SP). ESPÍRITO SANTO: Linhares, 6.XII.2007, D.A.Folli 5796 (CVRD). MINAS GERIAS: Dionísio, 7.V.2003, G.S.França 323 (HUEFS); PARANÁ: Ribeirão do Pinhal, 12.XI.2000, J.Carneiro 806 (RB). RIO DE JANEIRO: Itatiaia, 23.III.2002, P.P.de Souza 147 (RB). RIO GRANDE DO SUL: Três Forquilhas e Três Cachoeiras, 23.II.2003, B.E.Diaz 612 (R). SÃO PAULO: Parque Estadual Turístico Alto Ribeira, 25.II.2011, L.C.Pederneiras et al. 652 (SP).

2.5 Ficus gomelleira Kunth emend. Carauta \& Diaz Fig. $4 \mathrm{a}$

Extensão de ocorrência: ca. 5 milhões de $\mathrm{km}^{2}$. Material selecionado: ALAGOAS: Ibateguara, 16.X.2002, M.Oliveira et al. 1128 (R). AMAPÁ: Rio Vila Nova, 13.VII.1951, R.L.Froes \& Black (R 27482). AMAZONAS: São Sebastião do Uatumã, 13.VII.1979, C.A.Cid et al. 310 (R). BAHIA: Ibicoara, 13.X.2007, M.L.Guedes et al. 13895 (ALCB); CEARÁ: F.Alemão 1434 (R). ESPÍRITO SANTO: Iporá, 16.IV.2005, L.P.Queiroz et al. 10337 (HUEFS). MARANHÃO: Viana e Bandeirantes, 17.X.1980, D.C.Daly et al. D634 (HRB). MATO GROSSO: Sinop, 3.X.1985, Cid Ferreira et al. 6323 (SPF). MINAS GERAIS: Governador Valadares, 23.II.2004, R.M.Castro \& L.Teixeira 916 (HUEFS). PARAÍBA: João Pessoa, 2.VIII.2001, P.C.Gadelha Neto et al 659 (R). PIAUÍ: Caracol, 22.VI.2007, R.M.Santos et al. 1442 (HUEFS). RIO DE JANEIRO: Cabo Frio, 30.X.2008, L.C.Pederneiras \& R.W.Lacerda 509 (R). Itatiaia, 23.III.2002, P.P.Souza 144 (R). RIO GRANDE DO SUL: Tramandaí, 3.III.1985, J.R.Stehmann (GUA 43587). RONDÔNIA: Costa Marques para Forte Principe da Beira, 28.III.1987, M.Nee 34522 (RB). SANTA 


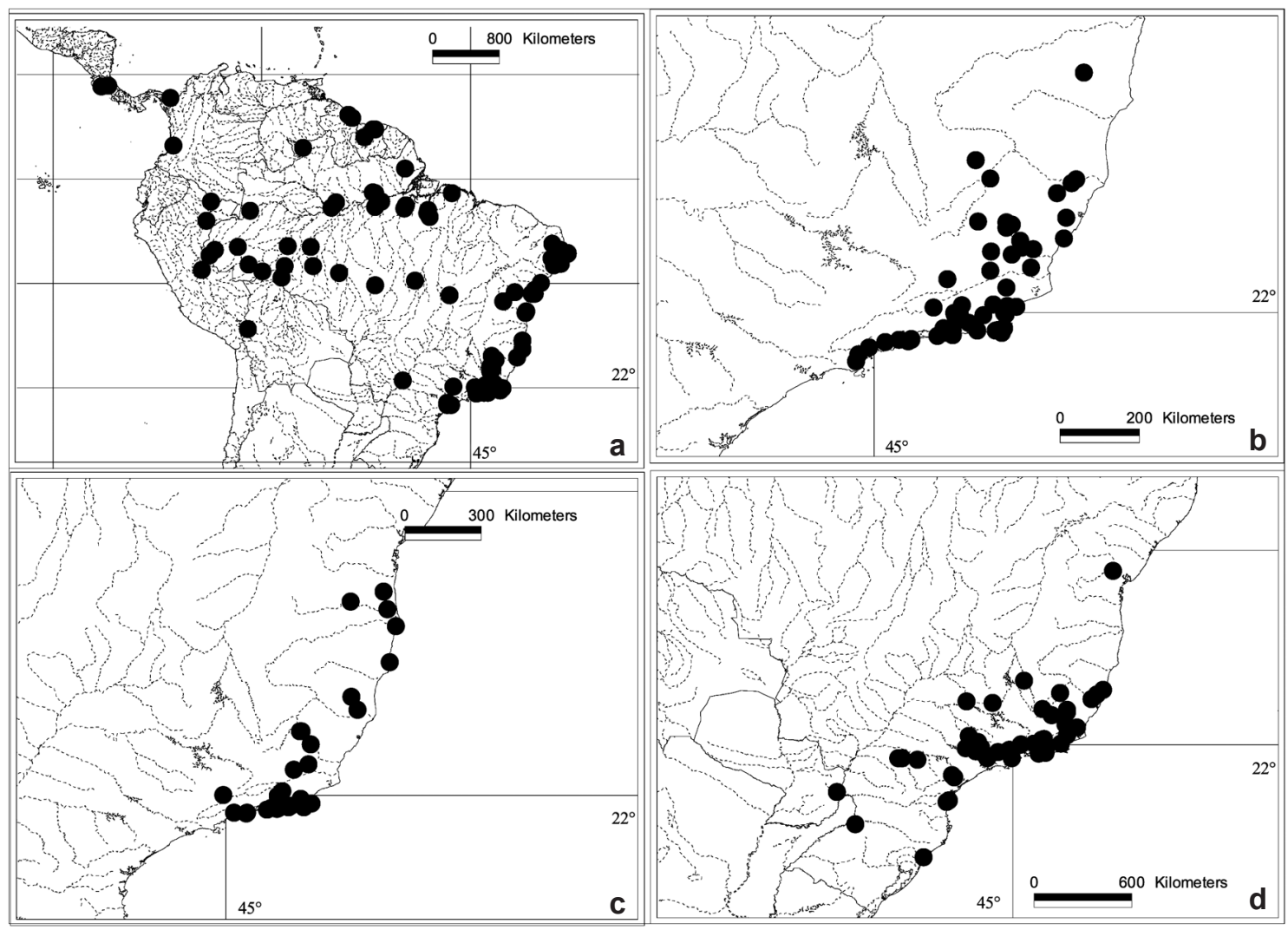

Figura 3 - Mapas de distribuição geográfica - a. Brosimum guianense; b. Dorstenia arifolia; c. Dorstenia cayapia; d. Ficus eximia.

Figure 3 - Geographic distribution maps - a. Brosimum guianense; b. Dorstenia arifolia; c. Dorstenia cayapia; d. Ficus eximia.

CATARINA: Itajaí, 14.XII.1975, Klein 11261 (GUA). SÃO PAULO: Areias, 11.IV.2005, L.R.Mendonça-Souza et al. 35 (RB).

\subsection{Ficus hirsuta Schott}

Fig. $4 b$

Extensão de ocorrência: ca. 340 mil km².

Materiais selecionados: BAHIA: Almadina, 19.III.2006, J.L.Paixão et al. 862 (HUEFS). ESPÍRITO SANTO: entre Vitória e Guarapari, 19.VIII.2003, B.E.Diaz 690 (R). MATO GROSSO: Pantanal, 13.VII.1995, M.R.PereiraNoronha 1131 (SP). MINAS GERAIS: Barroso, Mata do Baú, 28.IX.2002, L.C.S.Assis \& M.S.Magalhães 580 (SP). RIO DE JANEIRO: Saquarema, 17.I.2008, L.C.Pederneiras \& A.F.P.Machado 355 (R). SÃO PAULO: Gália, Estação Ecológica dos Caetetus, 12.III.1997, E.Furlan (SP 396897).

\subsection{Ficus organensis (Miq.) Miq.}

Fig. 4c

Extensão de ocorrência: ca. 240 mil km².

Materiais selecionados: BAHIA: Salvador, 18.II.1992, M.L.Guedes et al. 2569 (R). Sauípe, 15.XI.2007, E.P.Queiroz 2513 (HRB). ESPÍRITO SANTO: Alfredo Chaves, 7.VII.1996, G.Hatschbach et al. 65244
(HUEFS). MINAS GERAIS: Diamantina, 29.I.2000, R.Mello-Silva \& Forzza 1741 (SPF). PARANÁ: Doutor Ulysses, 2.XII.1999, G.Hatschbach et al. 69808 (RB). PERNAMBUCO: Floresta, Inajá, 4.VI.1995, M.C.Tschá et al. 113 (RB). RIO DE JANEIRO: Macaé, 16.VII.2005, L.C.Pederneiras 184 (R). RIO GRANDE DO SUL: Gravataí, 27.II.2003, B.E.Diaz 1431 (R). SANTA CATARINA: Blumenau, 17.III.1997, C.E.Zimmermann (GUA44916). SÃO PAULO: Caraguatatuba, 16.III.2003, A.Oliveira et al. 2103 (RB).

2.8 Ficus pulchella Schott ex Spreng. Fig. 4d Extensão de ocorrência: ca. $200 \mathrm{mil} \mathrm{km²}$.

Material selecionado: BAHIA: Itaberaba, 18.I.2006, L.P.Queiroz et al. 12058 (HUEFS). ESPÍRITO SANTO: Conceição da Barra, 22.IX.1992, O.J.Pereira 3885 (GUA). MINAS GERAIS: Caratinga, 11.VII.1980, A.Nishimura 55 (GUA). PARAÍBA: Espírito Santo, 25.XI.1968, Andrade-Lima 5479 (SP). RIO DE JANEIRO: Arraial do Cabo, 28.VIII.1996, L.E.MelloFilho et al. 6102 (R). SANTA CATARINA: Cubatão, 21.VI.1986, S.L.Pompéia 1 (SP). SÃO PAULO: Peruibe, 30.X.2010, L.C.Pederneiras et al. 589 (SP). 


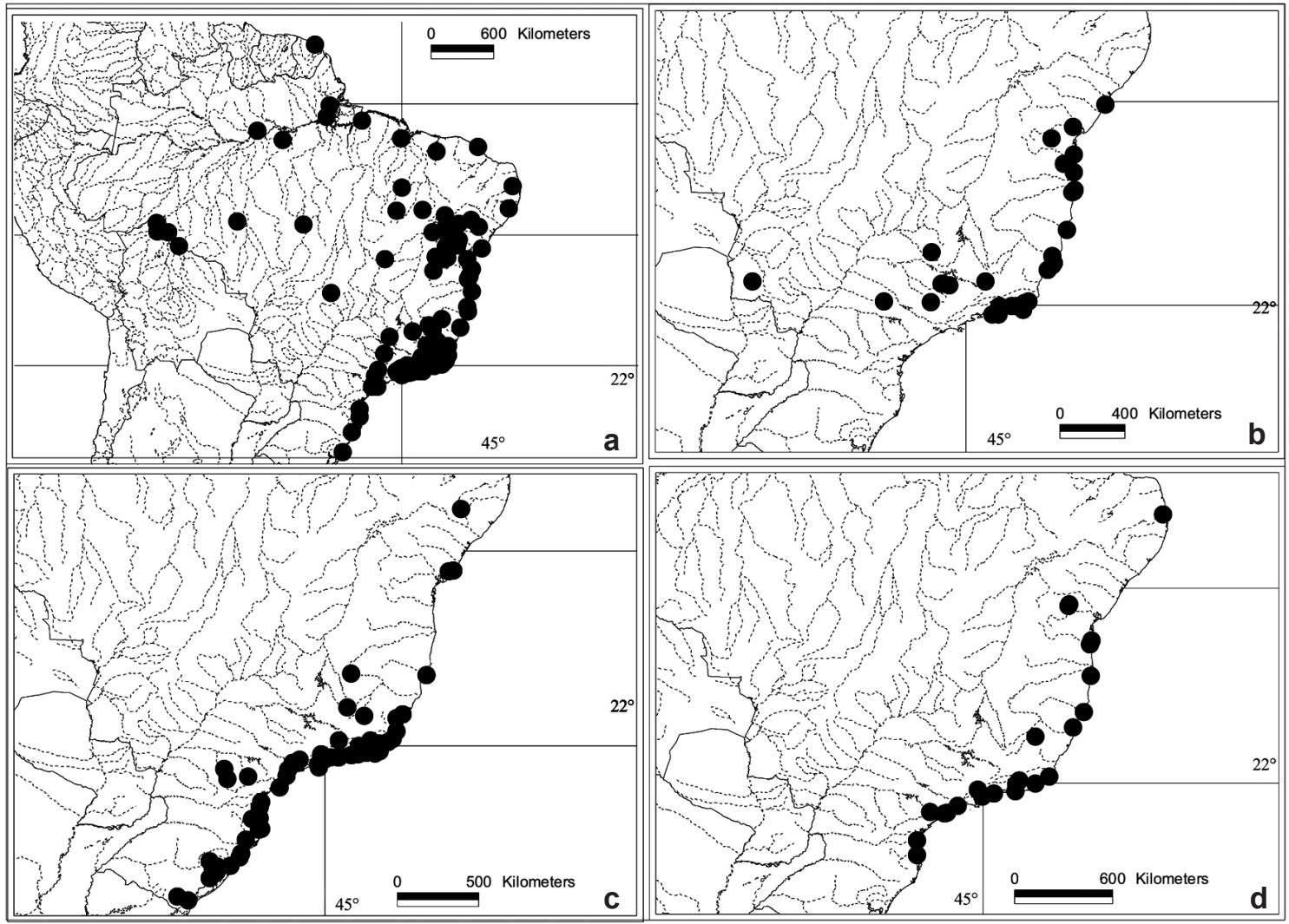

Figura 4-Mapas de distribuição geográfica-a. Ficus gomelleira; b. Ficus hirsuta; c. Ficus organensis; d. Ficus pulchella. Figure 4 - Geographic distribution maps - a. Ficus gomelleira; b. Ficus hirsuta; c. Ficus organensis; d. Ficus pulchella.

\subsection{Maclura tinctoria (L.) D. Don ex Steud.}

Fig. 5a

Extensão de ocorrência: ca. 11,5 milhões de $\mathrm{km}^{2}$.

Material selecionado: ACRE: $11 \mathrm{~km}$ de Rio BrancoQuixada, 18.X.1980, S.R.Lowrie et al. 576 (R). BAHIA: Anguera, 22.V.2007, D.Cardoso \& R.M.Santos 1947 (HUEFS). BRASILIA: 2.X.1992, B.A.S.Pereira \& V.V.Mecenas 2264 (RB). CEARÁ: Aiuaba, 25.II.1997, L.W.Lima-Verde et al. 455 (RB). ESPÍRITO SANTO: Guarapari, 25.III.1993, P.E.Vinha (SP 305557). GOIÁS: Luziania, 9.XI.2002, G.Pereira-Silva et al. 6993 (R). MARANHÃO: São Luis, 2.XI.1982, Gottsberger 21182 (SP). MATO GROSSO: Gaúcha do Norte, 5.X.2000, N.M.Ivanauskas 4405 (SP). MATO GROSSO DO SUL: Campo Grande, 15.X.1989, M.C.A.Fubenda et al. 64 (RB). MINAS GERAIS: Governador Valadares, 22.II.2004, R.M.Castro \& L.Teixeira 908 (HUEFS). PARAÍBA: João Pessoa, 8.III.1988, C.A.B.Miranda \& Moura 509 (SP). PARANÁ: Antonina, 10.IV.2003, M.Borgo et al. 2373 (RB). PERNAMBUCO: Cabo de Santo Agostinho, 30.IX.2007, E.P.Queiroz et el. 2468 (HRB). RIO DE JANEIRO: Cabo Frio, Estação de Rádio da Marinha,
29.X.2008, L.C.Pederneiras \& R.W.Lacerda 487 (R). RIO GRANDE DO SUL: São Leopoldo, XII.1994, J.Dutra (R 56304). RONDÔNIA: 18.I.1979, G.Vieira et al. 530 (R). SANTA CATARINA: Palhoça, 24.VI.1971, R.M.Klein 9543 (GUA). SÃO PAULO: Eldorado, 28.III.2005, J.E.Meireles et al. 291 (SP). SERGIPE: Carmópolis, 23.IV.2000, A.M.Teles 9 (HRB). TOCANTINS: São Sebastião do Tocantins, 13.XI.1983, E.Mileski 334 (HRB).

2.10 Sorocea guilleminiana Gaudich. Fig. $5 b$ Extensão de ocorrência: ca. 2,2 milhões de km² . Material selecionado: BAHIA: Itaberaba, 18.I.2006, L.P.Queiroz 12057 (HUEFS). BRASÍLIA: 14.IX.1984, B.A.S.Pereira 1184 (SP). ESPÍRITO SANTO: Santa Teresa, 2.XII.1987, W.Pizziolo 332 (GUA). GOIÁS: Cavalcante, 18.X.2001, G.Pereira-Silva et al. 5700 (SP). MINAS GERAIS: Marliéria, 6.X.2003, G.S.França 417 (HUEFS). PARAÍBA: Sapé, 23.III.2001, G.O.Dionisio 109 (R). PERNAMBUCO: Ipojuca, 6.IX.1967, P.Ferreira 67-28 (SP). RIO DE JANEIRO: Cabo Frio, 2.IV.2008, L.C.Pederneiras \& M.S.Faria 398 (R). SÃO PAULO: Ubatuba, 5.XII.1988, F.C.P.Garcis et al. 319 (SP). 

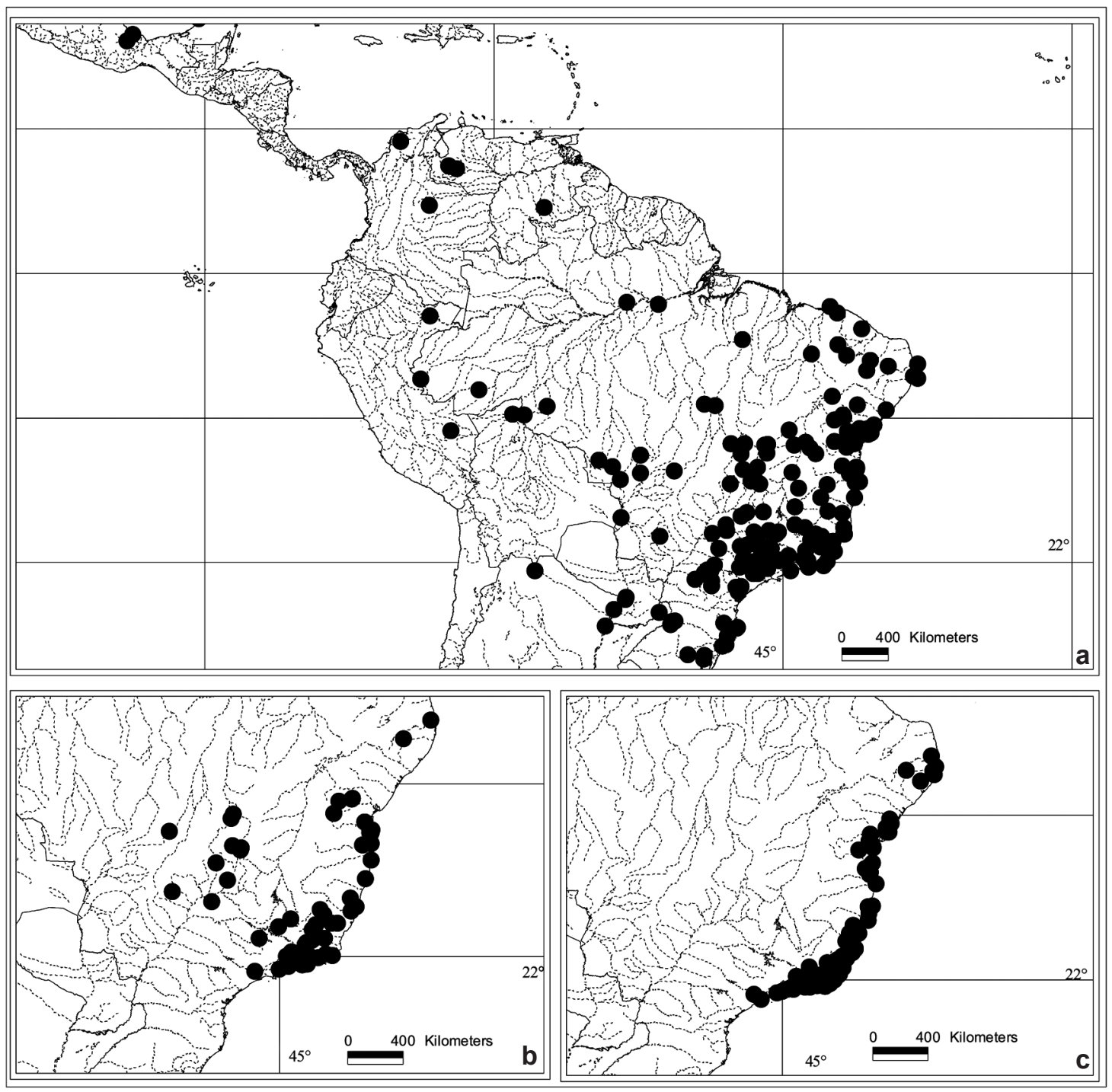

Figura 5 - Mapas de distribuição geográfica - a. Maclura tinctoria; b. Sorocea guilleminiana; c. Sorocea hilarii. Figure 5 - Geographic distribution maps - a. Maclura tinctoria; b. Sorocea guilleminiana; c. Sorocea hilarii.

\subsection{Sorocea hilarii Gaudich.}

Extensão de ocorrência: ca. 220 mil km². Material selecionado: ALAGOAS: Ibateguara, 3.IV.2003, M.Oliveira \& Grillo 1324 (HUEFS). BAHIA: Ituberá, 8.IV.2006, R.M.Valadão \& Guedes 261 (ALCB).ESPÍRITO SANTO: Itapemirim, 21.VI.1996, R.Mello-Silva et al. 1184 (SP). MINAS GERIAS: Coronel Pacheco, 27.XI.1944, Ezequieu 1660 (SP). PERNAMBUCO: Sirinhaém, 11.II.2004, M.Oliveira \& Grillo 1539 (R). RIO DE JANEIRO: São João da Barra, 1.XI.2008, L.C.Pederneiras \& R.W.Lacerda 544 (R). SÃO PAULO: São Sebastião, 21.IV.2000, J.P.Souza et al. 3342 (SP).
Espécies não ameaçadas de extinção

Todas as espécies a seguir são avaliadas como de menor preocupação (LC) porque são reconhecidas em diversas localidades, com coletas relativamente recentes, e possuem extensão de ocorrência maior que $20.000 \mathrm{~km}^{2}$.

\subsection{Cecropia glaziovii Snethl.}

Materiais selecionados: BAHIA: Amargosa, 24.I.2007, D.Cardoso 1525 (SP). ESPÍRITO SANTO: Domingos Martins, 29.V.1993, J.M.Simões et al. 73 (GUA). MINAS GERAIS: Monte Belo, 23.V.1987, H.C.Weyland Vieira 
1194 (R). PARANÁ: Cerro Azul, 8.II.1996, E.V.Odia et al. 51 (RB). RIO DE JANEIRO: Cabo Frio, 30.X.2008, L.C.Pederneiras \& R.W.Lacerda 516 (R). RIO GRANDE DO SUL: Osório, 22.XII.1979, Waechter \& Baptista 1516 (GUA). SANTA CATARINA: Reserva Vila Velha, 17.II.1993, Negrelle \& Londero 743 (GUA). SÃO PAULO: Apiaí, 10.I.1993, S.Romaniuc-Neto et al. 1388 (SP).

\subsection{Cecropia lyratiloba Miq.}

Material selecionado: BAHIA: Lauro de Freitas, 15.III.2006, E.P.Queiroz \& Queiroz 1720 (HRB). BRASÍLIA: Distrito Federal, 19.V.1978, Heringer et al. 509 (GUA). ESPÍRITO SANTO: Retiro Aracruz, 6.V.1992, O.Pereira et al. 3394 (GUA). GOIÁS: Campinaçu, 6.IX.1995, B.Walter et al. 2556 (GUA). MINAS GERIAS: Januária, 12.IX.2003, M.G.Bovine 2303 (RB). PARANÁ: Rio Piquiri, 3.II.1982, P.P.Furtado 154 (RB). PERNAMBUCO: Cabo de Santo Agostinho, 12.XI.2007, E.P.Queiroz \& Queiroz 2491 (HRB). RIO DE JANEIRO: Cabo Frio, 29.X.2008, L.C.Pederneiras \& R.W.Lacerda 499 (R). SÃO PAULO: Cananéia, 11.II.1992, S.Romaniuc-Neto \& Godoi 1280 (SP). SERGIPE: Areia Branca, 23.I.1992, J.P.P.Carauta 6484 (GUA).

3.3 Celtis brasiliensis (Gardner) Planch.

Material selecionado: BAHIA: Abaíra, 22.I.1994, W. Ganev 2858 (SPF). BRASILIA: 15.IX.1981, B.A.S.Pereira 58 (RB). GOIÁS: Serra do Rio Preto, leste de Cabeceiras, 16.XI.1965, Irwin et al. 10348 (GUA). MATO GROSSO: Cuiabá, X.1914, J.G.Kuhlmann 162 (R). MATO GROSSO DO SUL: Bonito, 10.X.2003, G.Hatschbach et al. 76106 (RB). MINAS GERAIS: Serra de São José, 6.X.1997, M.C.Vianna et al. 2432 (GUA). RIO DE JANEIRO: Mangaratiba, Restinga de Marambaia, 6.V.2008, L.C.Pederneiras et al. 428, 429 (R). SANTA CATARINA: Bom Retiro, Pinheiral, 24.XI.1956, L.B.Smith \& Klein 7923 (R). SÃO PAULO: Votorantin, 20.III.1983, V.F.Ferreira 3007 (GUA).

\subsection{Celtis iguanaea (Jacq.) Sarg.}

Material selecionado: BAHIA: Iaçu, Lage Preta, 12.III.2005, França et al 5150 (SP). CEARÁ: Pacatuba, 18.V.1935, F.E.Drouet 2626 (R). ESPÍRITO SANTO: Linhares, 25.III.1987, Folli 636(GUA). GOIÁS: Monte Alegre, 11.IV.2000, R.C.Mendonça et al. 4147 (GUA). MARANHÃO: Vitória do Mearim, 26.XI.1985, J.G.Silva 2027 (R). MATO GROSSO: Santa Teresinha, 13.X.1985, W.Thomas et al. 4371 (SPF). MINAS GERAIS: Caxambu, 20.II.2006, H.C.L.Geraldo 313 (HB). PARÁ: Arapari, Monte Alegre, 27.IX.1953, Froés 30201 (R). PARANÁ: Sapopema, 19.IV.1997, V.F.Kinnup et al. 432 (SPF). PARAÍBA: estrada Tabaiana-Pilar, 25.VIII.1952, A.Lima 1168 (R). PERNAMBUCO: Triunfo, 18.VI.1992, E.Ferraz 164 (SP). RIO DE JANEIRO: Cabo Frio, 29.X.2008, L.C.Pederneiras \& R.W.Lacerda 484 (R). RIO GRANDE DO SUL: Esmeralda, 11.XI.1984, Jarenkow 152 (RB). SANTA CATARINA: Anita
Garibaldi, 12.IV.1963, Reitz \& Klein 14758 (R). SÃO PAULO: Pedregulho, 6.IV.2004, A.B.Junqueira \& D.Sasaki 190 (SPF).

3.5 Coussapoa microcarpa (Schott) Rizzini Material selecionado: BAHIA: Eunápolis, 6.XII.2004, R.M.Castro 1067 (HUEFS). ESPÍRITO SANTO: Pinheiros, 11.VIII.2004, L.S.Leoni 5932 (R). MINAS GERAIS: Barroso, 25.XI.2001, L.Assis \& Ladeira 392 (GUA). PARANÁ: Antonina, 12.I.2006, O.S.Ribas et al. 7168 (ALCB). PERNAMBUCO: Bonito, 28.I.1970, Andrede-Lima 5668 (RB). RIO DE JANEIRO: Angra dos Reis, IV.1996, L.E.Mello-Filho et al. 5835 (R). RIO GRANDE DO SUL: Montenegro, 30.XI.1988, I.Fernandes 433 (GUA). SANTA CATARINA: Itapoá, 13.X.1992, R.Negrelle A-467 (GUA). SÃO PAULO: São José dos Campos, XI.2002, M.A.Assis 1654 (RB).

\subsection{Ficus arpazusa Casar.}

Material selecionado: ALAGOAS: $31 \mathrm{~km}$ de Jamarataia, 9.VI.1981, Andrade-Lima et al. 45 (SP). AMAZONAS: Reserva de Desenvolvimento Sustentável Mamirauá, 6.V.2000, M.R.Mesquita 293 (HRB). BAHIA: Amargosa, 4.VIII.2007, J.L.Paixão \& Nascimento 1315 (HUEFS). BRASÍLIA: 2.X.1978, E.P.Heringer 16108 (RB). ESPÍRITO SANTO: Vila Velha, 11.IV.1996, Zambom \& Fernades 270 (SP). GOIÁS: Almadina, 21.VI.2005, P.Fiaschi et al. 2913 (SPF). MARANHÃO: São Luis, 15.IV.1993, F.H.Muniz 181 (RB). MATO GROSSO DO SUL: Bataipora, 26.III.1986, U.Pastore \& Klein 70 (HRB). MINAS GERAIS: Santa Maria do Salto, 22.IV.2006, A.M.Amorim et al. 5896 (HUEFS). PARÁ: São Geraldo do Araguaia, 22.IV.2004, G.Pereira-Silva et al. 8961 (RB). PARAÍBA: Lagoa Seca, Fazenda Ipuarana, 29.VII.2001, C.E.Lourenço et al. 240 (R). PARANÁ: Jundiaí do Sul, 15.III.1996, J.Carneiro 202 (R). RIO DE JANEIRO: Macaé, 31.X.2008, L.C.Pederneiras \& R.W.Lacerda 536 (R). RONDÔNIA: Aríquemes, 16.V.1982, Teixeira et al. 531 (RB). SANTA CATARINA: Paulo Lopes, 23.VI.1971, Klein 9535 (GUA). SÃO PAULO: São Vicente, 15.II.2002, Pastore \& Moura 1121 (SP).

\subsection{Ficus castellviana Dugand}

Material selecionado: AMAZÔNIA: Presidente Figueiredo, Mineração Taboca, 11.X.1979, G. Vieira et al. 385 (INPA). BAHIA: Arataca, 17.III.2005, P.Fiaschi et al. 2811 (SPF). ESPÍRITO SANTO: Linhares, 13.I.1993, D.A.Folli 1792 (GUA). MINAS GERAIS: Caratinga, Descoberto, 2.II.2000, L.D.Meireles (GUA 48387). RIO DE JANEIRO: Cabo Frio, Parque Estadual do Mico Leão Dourado, 30.X.2008, L.C.Pederneiras \& R.W.Lacerda $505,507,529(\mathrm{R})$.

3.8 Ficus clusiifolia (Miq.) Schott Material selecionado: BAHIA: Salvador, 31.I.2006, G.M.Carvalho \& Roque 73 (ALCB). ESPÍRITO SANTO: Santa Leopoldina, 21.I.2006, M.O.S.Crepaldi 
et al. 73 (RB). PERNAMBUCO: São Vicente Férrer, 1.IV.1996, M.F.A.Lucena et al. 449 (GUA). RIO DE JANEIRO: Carapebus, 18.X.2007, L.C.Pederneiras et al. $322(\mathrm{R})$.

3.9 Ficus luschnathiana (Miq.) Miq. Material selecionado: BAHIA: Porto Seguro, 4.VII.1990, Folli 1190 (GUA). ESPÍRITO SANTO: Conceição da Barra, 5.XI.1992, O.J.Pereira et al. 4145 (GUA). MATO GROSSO: Rio Branco, 10.V.1995, C.Haschbach (RB 62616). MATO GROSSO DO SUL: Amambai, 28.I.1979, J.P.P.Carauta 3079 (RB). MINAS GERAIS: Rio Preto, 25.IV.2004, K.Antunes et al. 113 (HUEFS). PARANÁ: Adrianópolis, 15.IX.1999, J.M.Cruz et al. 189 (RB). RIO DE JANEIRO: Cabo Frio, 30.X.2008, L.C.Pederneiras \& R.W.Lacerda 511 (R). RIO GRANDE DO SUL: Pessegueiro, Camaquã, 11.X.1983, M.Sobral 2275 (RB). SANTA CATARINA: Campo Alegre, 17.I.1996, O.S.Ribas et al. 984 (GUA). SÃO PAULO: Agudos, 1.VII.2005, L.R.Mendonça-Souza \& Salvador 50 (SP).

3.10 Ficus maximiliana (Miq.) Mart.

Material selecionado: ESPÍRITO SANTO: Linhares, 17.I.1996, D.A.Folli 2684 (GUA). RIO DE JANEIRO: Saquarema, 17.I.2008, L.C.Pederneiras \& A.F.P.Machado $356(\mathrm{R})$.

\subsection{Ficus trigona L.f.}

Material selecionado: ACRE: H. Medeiros 637 (UFACPZ). AMAPÁ: Estrada do Igarapé Pacoval, NE de Macapá, 13.XI.1979, C.Rosário \& Santos 7388 (GUA). AMAZONAS: Reserva de Mamirauã, 6.V.2000, M.R.Mesquita 294 (HRB). BAHIA: Barro Preto, 13.XII.2004, R.M.Castro 12 (HUEFS). BRASÍLIA: 21.IX.1993, B.A.S.Pereira \& Alvarenga 2568 (GUA). ESPÍRITO SANTO: Linhares, 28.II.1996, Folli 2694 (GUA). GOIÁS: Nerópolis, 1.IX.2005, B.A.S.Pereira et al. 3532 (RB). MARANHÃO: Carolina, 15.IV.1983, M.F.F. Silva et al. 1103 (HRB). MATO GROSSO: Porto Esperidião, 18.XI.1996, Hatschbach et al. 65651 (R). MATO GROSSO DO SUL: Santa Rita do Pardo, 14.X.1998, A.Amaral et al. 192 (SP). MINAS GERAIS: Araguari, 30.I.1993, G.M.Araujo et al. 929 (SP). Faria Lemos, 30.X.2001, L.S.Leoni 4779 (R). PARÁ: Marabá, 29.V.1982, R.Sacco et al. 390 (RB). PARANÁ: Ponta Grossa, 3.VII.1970, Krieger 8770 (GUA). RIO DE JANEIRO: Cabo Frio, 2.IV.2008, L.C.Pederneiras \& M.S.Faria 402 (R). RONDÔNIA: Ariquemes, 16.V.1982, L.O.A.Teixeira et al. 527 (RB). RORAIMA: Rio Branco, abaixo do Rio Anauá, 1.V.1974, J.M.Pires et al. (RB 176157). SÃO PAULO: Agudos, 31.V.2000, Paschoal 2241 (SP).

\subsection{Laportea aestuans (L.) Chew}

Material selecionado: ALAGOAS: Pão de Açucar, 23.VI.2002, R.P.Lyra-Lemos 6925 (HUEFS). AMAZONAS: Anguera, 31.X.2006, J.S.Novais et al.
33 (HUEFS). Quijingue, 8.VII.2006, D.Cardoso 1335 (HUEFS). CEARÁ: Cedro, 23.VI.1912, Loefgren 1078 (R). ESPÍRITO SANTO: Linhares, 27.III.1971, T.S.Santos 1492 (CEPEC). MINAS GERAIS: Viçosa, 1.XII.1964, W.N.Vidal 255 (GUA). PARÁ: Conceição do Araguaia, 8.II.1980, T.Plowman et al. 8499 (HRB). PERNAMBUCO: Primavera, 25.V.1992, R.Pereira et al. (SP 305464). PIAUÍ: Amarante, 21.III.1984, S.B.Silva \& Faria 333 (HRB). RIO DE JANEIRO: Cabo Frio, 9.VI.2008, L.C.Pederneiras et al. 464 (R). RIO GRANDE DO SUL: Canguaretama, 5.VII.1959, L.E.Mello-Filho 1764 (R); SÃO PAULO: São Paulo, 17.VIII.2005, M.D.M.Vianna-Filho 750 (R).

\subsection{Trema micrantha (L.) Blume}

Material selecionado: ACRE: Cruzeiro do Sul, Rio Moa, Igarapé São Francisco, 9.V.1971, Maas et al. 12827 (R). ALAGOAS: Ibateguará, 15.VI.2002, M.Oliveira \& Grilo 977 (HUEFS). AMAPÁ: Porto de Santana, 10.II.1960, J.P.P.Carauta 137 (R). AMAZONAS: Manaus, Reserva Florestal Ducke, 17.IV.1997, M.A.D.Souza et al. 352 (SP). BAHIA: Alagoinhas, 5.XI.2002, M.R.Fonseca 570 (ALCB). CEARÁ: Cratéus, 5.VI.2002, F.S.Araujo 1575 (HUEFS). ESPÍRITO SANTO: Anchieta, 10.V.1988, J.Gomes 586 (SP); Colatina, 28.I.1997, M.Arbor et al. 7757 (CEPEC). GOIÁS: Campinaçu, 25.X.1995, Walter et al. 2862 (SP). MARANHÃO: São Luis, 9.VI.1992, Muniz 160 (RB). MATO GROSSO: Cáceres, 23.XI.1984, Emmerich et al. 5275 (R). MATO GROSSO DO SUL: Três Lagoas, 7.IV.1999, A.Amaral et al. 307 (ALCB). MINAS GERAIS: Descoberto, 2.XII.2001, R.M.Castro 729 (GUA). PARÁ: Altamira, 30.X.1977, A.S.Silva et al. 198 (RB). PARAÍBA: Pedras de Fogo, 7.III.2001, Bayma 659 (HUEFS). PARANÁ: Londrina, XII.2002, Estevan et al. 366 (RB). PERNAMBUCO: Brejo da Madre de Deus, 18.X.1999, Nascimento 264 (CEPEC). PIAUÍ: Teresina, 18.III.2006, Pederneiras \& Carvalho 204 (R). RIO DE JANEIRO: Cabo Frio, Parque Municipal do Mico Leão Dourado, 30.X.2008, L.C.Pederneiras \& R.W.Lacerda 510 (R). RIO GRANDE DO NORTE: Natal, 6.XI.1980, s/c 225 (R). RIO GRANDE DO SUL: Belém Novo, 18.I.1964, Castellanos 24476 (GUA). RONDÔNIA: Ariquemes, 12.V.1982, L.O.A.Teixeira et al. 368 (RB). RORAIMA: Caracaraí, 15.VI.1985, I.Cordeiro et al. 51 (SP). SANTA CATARINA: Concórdia, 10.IX.1986, J.A.F.Costa 66 (R). SÃO PAULO: Luiz Antonio, 7.X.1999, Nicolau \& Faria 1935 (SP). TOCANTINS: Esperantina, 30.XI.1999, Pedro 5 (HUEFS).

\subsection{Urera aurantiaca Wedd.}

Material selecionado: ACRE: Rio Branco, 29.XI.1993, C.Figueiredo 126 (INPA). AMAZÔNIA: Carauari, Marapata, 25.V.1933, B.A.Krukoff 4568 (F). BAHIA: Camacã, 9.IV.1965, M.Magalhães 19573 (HB). MARANHÃO: Açailândia, 17.XII.1978, N.A.Rosa \& Vilar 3029 (NY). MATO GROSSO DO SUL: Porto da Esperança, VIII.1927, Luetzelburg 21442 (R). MINAS 
GERAIS: Matozinho, 27.III.1995, A.E.Brina \& Costa (RB 409183). PARÁ: Arumanduba, 27.VII.1961, W.A.Egler \& Irwin 46036 (R). PARANÁ: Foz do Iguaçu, 17.II.1960, E.Pereira 5337 (HB). RIO DE JANEIRO: Cabo Frio, 24.IV.1979, J.P.P.Carauta 3098 (RB); Silva Jardim, 18.IV.1977, J.P.P.Carauta 2422 (RB). SANTA CATARINA: Itajaí, 8.II.1955, R.Klein 1129 (HB); Mondaí, 31.XII.1963, Reitz 16691 et Klein (R). SÃO PAULO: Iporanga, 22.XII.2010, L.C.Pederneiras 665 et al. (SP).

3.15 Urera nitida (Vell.) Brack.

Material selecionado: ACRE: Cruzeiro do Sul, 17.IV.1971, Prance et al. 12009 (R). ALAGOAS: Pão de Açucar, 10.VI.1981, Andrade-Lima et al. 69 (HRB). AMAZONAS: Reserva Indígena dos Yanomamis, VIII.1993, W.Milliken 1785 (INPA). BAHIA: Anguera, 21.XI.2006, D.Cardoso \& França 1423 (HUEFS). BRASILIA: 15.XII.1965, Irwin et al. 11317 (RB). CEARÁ: Serra de Baturite, Sitio Caridade, 10.XII.1937, Jose 480 (RB). ESPÍRITO SANTO: Santa Teresa, 8.V.1984, Boone 115 (CEPEC). MINAS GERAIS: Descoberto, 3.XI.2002, Assis et al. 607 (RB). PARANÁ: Campo Mourão, 13.IV.2007, M.G.Caxambu 1484 (RB). PERNAMBUCO: Brejo da Madre de Deus, 14.IX.1999, C.A.M.Oliveira et al. 22 (CEPEC). RIO DE JANEIRO: Cabo Frio, 29.X.2008, L.C.Pederneiras \& R.W.Lacerda 490 (R). Nova Friburgo, 23.IV.2004, A.F.P.Machado 173 (R). RIO GRANDE DO SUL: Irai, Balneário Oswaldo Cruz, 23.I.1990, A.Krapovickas \& Cristobal 43477 (F). SÃO PAULO: Iporanga, 22.XII.2010, L.C.Pederneiras et al. 654 (SP).

\section{Conclusões}

Dentre as 32 espécies de Urticineae ocorrentes nas restingas fluminenses, Ampelocera glabra, Celtis spinosa, Ficus cyclophylla, F. nevesiae, Maclura brasiliensis e Phyllostylon brasiliense encontraram-se ameaçadas de extinção. Estas espécies ocorrem, juntas ou separadas, atualmente em Armação de Búzios (Praia da Gorda, da Tartaruga e Tucuns), Arraial do Cabo (Massambaba), Cabo Frio (Parque Municipal do Mico Leão Dourado, Unamar, Peró), Rio de Janeiro (Morro da Babilônia, Morro da Urca e Prainha) e Saquarema (Jacarepiá e Jaconé), o que confere a estas localidades importância conservacionista.

Brosimum guianense, Dorstenia arifolia, Ficus pulchella e Sorocea guilleminiana em estudos anteriores foram consideradas em perigo, mas no presente trabalho foram reavaliadas e classificadas na categoria Menor Preocupação (LC). Essa mudança ocorreu devido a novas informações dos registros de herbários dos últimos 15 anos que indicaram coletas recentes, muitas dentro de Unidades de Conservação.

\section{Agradecimentos}

Ao Conselho Nacional de Pesquisa - CNPq a concessão de bolsa ao primeiro autor, e aos Departamentos de Botânica (Dra. Mariângela Menezes) e de Transporte (Sr. Miguel) do Museu Nacional da Universidade Federal do Rio de Janeiro.

\section{Referências}

Borges, R.; Moraes, M.A.; Monteiro, N.P.; Bevacqua, A.M.; Martinelli, G. \& Marquete, N. 2012. Available data and risk assessment of the Brazilian threatened species of Combretaceae. Rodriguésia 63: 031-038.

Carauta, J.P.P. 1996. Moraceae do estado do Rio de Janeiro. Albertoa 4: 145-194.

Carauta, J.P.P 2002. Ficus nevesiae Carauta (Moraceae) nova espécie e ameaçada de extinção. Albertoa. Série Urticineae 10: 65-67.

Carauta, J.P.P.; Sastre, C. \& Romaniuc-Neto, S. 1996. Índice de Moráceas do Brasil. Albertoa 4: 77-93.

CONABIO. 2009. Catálogo taxonómico de especies de México. 1. In: Capital Nat. México. CONABIO, Mexico City.

ENRI. 1999. ArcView (R) GIS 3.2a. New York: Environmental Systems Research Institute Inc.

IUCN Standards and Petitions Subcommittee. 1994. Red List Categories and Criteria version 2.3

IUCN Standards and Petitions Subcommittee. 2011 Guidelines for Using the IUCN Red List Categories and Criteria. Version 9.0. Prepared by the Standards and Petitions Subcommittee. Disponível em <http:// www.iucnredlist.org/documents/RedListGuidelines. pdf $>$. Acesso em 10 Out 2011.

IUCN Standards and Petitions Subcommittee. 2012. IUCN Red List of Threatened Species. Version 2012.1. Disponível em $<$ http://www.iucnredlist. org > . Acesso em 26 Jul 2012.

Judd, W.S.; Campbell, C.S.; Kellogg, E.A.; Stevens, P.F. \& Donoghue, M.J. 2007. Plant systematics: A phylogenetic approach. $3^{\text {rd }}$ ed. Sinauer, Sunderland.

Kaastra, R.C. 1973. Description and taxonomic position of Maclura brasiliensis (Moraceae). Acta Botanica Neerlandica 22: 69-74.

MMA Ministério do Meio Ambiente. 2008. Instrução Normativa No 6 de 23/09/2008.

Pederneiras, L.C. 2009. Urticales das restingas do estado do Rio de Janeiro: flora e padrões de distribuição geográfica. Dissertação de Mestrado. Universidade Federal do Rio de Janeiro, Rio de Janeiro. 166p.

Pederneiras, L.C.; Costa, A.F.; Araujo, D.S.D. \& Carauta, J.P.P. 2011a. Moraceae das restingas do estado do Rio de Janeiro. Rodriguésia 62: 77-92.

Pederneiras, L.C.; Costa, A.F.; Araujo, D.S.D. \& Carauta, J.P.P. 2011b. Ulmaceae, Cannabaceae e 
Urticaceae das restingas do estado do Rio de Janeiro. Rodriguésia 62: 299-313.

Pelissari, G. \& Romaniuc Neto, S. 2013. Ficus L. (Moraceae) da Serra da Mantiqueira. Rodriguésia 64: 91-111.

Romaniuc Neto, S.; Carauta, J.P.P.; Vianna Filho, M.D.M.; Pereira, R.A.S.; Ribeiro, J.E.L.S.; Machado, A.F.P.; Santos, A.; Pelissari, G. \& Pederneiras, L.C. 2012a. Moraceae. In: Lista de espécies da flora do Brasil. Jardim Botânico do Rio de Janeiro. Disponível em <http:// floradobrasil.jbrj.gov.br/2012/FB000167>. Acesso em Abr 2012.

Romaniuc Neto, S. \& Gaglioti, A.L. 2012. Urticaceae. In: Lista de espécies da flora do Brasil. Jardim Botânico do Rio de Janeiro. Disponível em < http:// floradobrasil.jbrj.gov.br/2012/FB000243>. Acesso em Abr 2012.
Romaniuc Neto, S. \& Torres, R.B. 2012. Ulmaceae. In: Lista de espécies da flora do Brasil. Jardim Botânico do Rio de Janeiro. Disponível em <http:// floradobrasil.jbrj.gov.br/2012/FB021357>. Acesso em Abr 2012.

Romaniuc Neto, S.; Torres, R.B. \& Santos, A. 2012 b. Cannabaceae. In: Lista de espécies da flora do Brasil. Jardim Botânico do Rio de Janeiro. Disponível em <http://floradobrasil.jbrj.gov. br/2012/FB106887>. Acesso em Abr 2012.

Sgrillo, R. 2012. Google Earth Path, v. 1.4.6.

Todzia, C. A. 1992. A reevaluation of the genus Phyllostylon (Ulmaceae). Sida 15: 263-270.

Todzia, C. A. 2001. Ulmaceae. In: Stevens, W.D.; Ulloa, C.; Pool, A. \& Montiel, O.M. (eds.). Flora de Nicaragua. Monographs in Systematic Botany from the Missouri Botanical Garden 85: 2472-2478. 